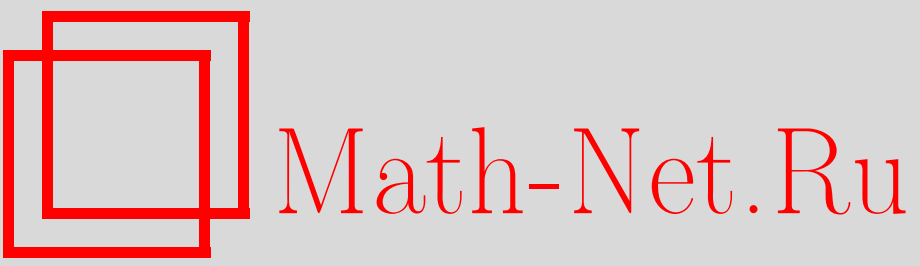

Я. Ю. Никитин, Р. С. Пусев, Точная асимптотика малых уклонений для ряда броуновских функционалов, Теория вероятн. и ее примен., 2012, том 57, выпуск $1,98-123$

DOI: https://doi.org/10.4213/tvp4433

Использование Общероссийского математического портала Math-Net.Ru подразумевает, что вы прочитали и согласны с пользовательским соглашением

http: //www . mathnet.ru/rus/agreement

Параметры загрузки:

IP : 54.166 .219 .16

26 апреля 2023 г., 16:20:43 
(C) 2012 г. НИКИТИН Я. Ю., ПУСЕВ Р. С.*

\title{
ТОЧНАЯ АСИМПТОТИКА МАЛЫХ УКЛОНЕНИЙ ДЛЯ РЯДА БРОУНОВСКИХ ФУНКЦИОНАЛОВ ${ }^{1)}$
}

\begin{abstract}
Найдена точная асимптотика малых уклонений по отношению к весовой гильбертовой норме для ряда хорошо известных гауссовских процессов. Используемый подход не требует знания собственных функций ковариационного оператора взвешенного процесса. Это позволяет обобщить многие ранее известные в этой области результаты. Попутно получены новые результаты о точной асимптотике малых уклонений для броуновской экскурсии, броуновского меандра, бесселевских процессов и мостов.

Ключевые слова и фразы: бесселевский процесс, броуновская экскурсия, броуновский меандр, гауссовский процесс, локальное время, малые уклонения.
\end{abstract}

1. Введение. Теория малых уклонений для гауссовских процессов интенсивно развивалась в последние годы (см. обзоры [47], [49], [18] и полную библиографию в [50]). Это развитие стимулировалось связями теории малых уклонений с рядом важных математических проблем, таких как точность дискретной аппроксимации для случайных процессов и проблема квантизации, вычисление метрической энтропии функциональных множеств или закон повторного логарифма в форме Чжуна. Было также обнаружено, что малые уклонения тесно связаны с функциональным анализом данных [36] и непараметрическим байесовским оцениванием [1], [60].

Проблема малых уклонений (называемая также проблемой малых шаров) для случайного процесса $X$ в норме $\|\cdot\|$ состоит в описании асимптотического поведения вероятности $\mathbf{P}\{\|X\| \leqslant \varepsilon\}$ при $\varepsilon \rightarrow 0$. Соотношение типа

$$
\mathbf{P}\{\|X\| \leqslant \varepsilon\} \sim C \varepsilon^{\beta} \exp \left(-d \varepsilon^{-\alpha}\right), \quad \varepsilon \rightarrow 0,
$$

${ }^{*}$ С.-Петербургский государственный университет, математико-механический факультет, Старый Петергоф, Университетский пр. 28, 198504 С.-Петербург, Россия; e-mail: yanikit47@gmail.com, ruslan.pusev@math.spbu.ru

1) Работа авторов была поддержана ФЦП «Научные и педагогические кадры инновационной России» (грант № 2010-1.1-111-128-033), грантом РФФИ № 10-01-00154 и программой поддержки ведущих научных школ (грант НШ-1216.2012.1). Р. С. Пусев был также поддержан Лабораторией им. П. Л. Чебышева СПбГУ, грант Правительства РФ дог. 11.G34.31.0026. 
с некоторыми вещественными константами $C, \beta, d$ и $\alpha$ называется точной асимптотикой. Менее точное утверждение вида

$$
\ln \mathbf{P}\{\|X\| \leqslant \varepsilon\} \sim-d \varepsilon^{-\alpha}, \quad \varepsilon \rightarrow 0
$$

называют логарифмической асимптотикой.

В известной монографии М.А.Лифшица $[7, \S 18]$ говорится: «Поведение малых уклонений, в отличие от больших, нельзя описать единообразно для всего класса гауссовских мер даже на логарифмическом уровне. Формализм оценивания значений малых уклонений, сравнимый по простоте с применением функционала действия для больших уклонений, еще не найден. Известны лишь частные результаты для нескольких важных ситуаций ...».

Случаи, когда в литературе о малых уклонениях удается выписать явные константы в точной и даже в логарифмической асимптотике, редки и относятся к небольшому числу простейших случайных процессов $([47],[18])$. В этой работе мы концентрируемся на задаче описания точной асимптотики малых уклонений в гильбертовой норме.

Пусть $X(t), a \leqslant t \leqslant b$, - центрированный гауссовский процесс с ковариационной функцией $G(t, s), a \leqslant t, s \leqslant b$. Для неотрицательной весовой функции $\psi(t)$, определенной на $[a, b]$, положим

$$
\|X\|_{\psi}=\left(\int_{a}^{b} X^{2}(t) \psi(t) d t\right)^{1 / 2}
$$

Если интеграл $\int_{a}^{b} G(t, t) \psi(t) d t$ конечен, то процесс $X(t) \sqrt{\psi(t)}$ допускает разложение Кархунена-Лоэва (см., например, [23]):

$$
X(t) \sqrt{\psi(t)}=\sum_{k=1}^{\infty} \xi_{k} \sqrt{\lambda_{k}} f_{k}(t)
$$

где $\xi_{k}, k \in \mathbf{N}$, независимые стандартные нормальные величины, тогда как $\lambda_{k}>0$ и $f_{k}(t), k \in \mathbf{N}$ - это собственные числа и собственные функции интегрального уравнения Фредгольма

$$
\lambda f(t)=\int_{a}^{b} G(t, s) \sqrt{\psi(t) \psi(s)} f(s) d s, \quad t \in[a, b] .
$$

Из разложения Кархунена-Лоэва следует, что

$$
\|X\|_{\psi}^{2}=\int_{a}^{b} X^{2}(t) \psi(t) d t \stackrel{\text { law }}{=} \sum_{k=1}^{\infty} \lambda_{k} \xi_{k}^{2}
$$

Поэтому исходная задача о малых уклонениях сводится к асимптотическому анализу вероятности $\mathbf{P}\left\{\sum_{k=1}^{\infty} \lambda_{k} \xi_{k}^{2} \leqslant \varepsilon^{2}\right\}$ при $\varepsilon \rightarrow 0$. Первое 
решение этой задачи было найдено Сытой [16], см. также обзоры [47] и [49]. Однако неявный характер решения, полученного в [16], делает невозможным извлечение из него точной асимптотики. Начиная с работ [6] и [34], многие авторы стремились упростить асимптотическое выражение для вероятности малых уклонений при тех или иных условиях.

Золотарев [62] получил точную асимптотику вероятности малых уклонений в случае $\lambda_{k}=k^{-A}, A>1$. Опираясь на результаты [48], Дункер, Лифшиц и Линде [35] нашли указанную асимптотику в случае $\lambda_{k}=f(k)$, где $f$ положительная, логарифмически выпуклая и дважды дифференцируемая суммируемая функция. В статье [27] оценки из [35] были применены к проинтегрированным и центрированным (по времени) броуновскому движению и броуновскому мосту. Более общие результаты для многократно проинтегрированных процессов были позже получены в [39] и [53]. В [11] точная асимптотика в рассматриваемой задаче была найдена в специальном случае, когда собственные числа $\lambda_{k}$ являются отношениями степеней двух полиномов по $k$.

Новый подход, позволяющий вычислить точную асимптотику малых уклонений в $L_{2}$-норме с точностью до константы, был разработан в [53] и [54] и применен к широкому классу гауссовских процессов, ковариации которых совпадают с функциями Грина самосопряженных дифференциальных операторов общего вида. Мы предлагаем называть такие процессы «процессами Грина».

Малые уклонения для взвешенных процессов Грина изучались в [10], где для достаточно гладких и невырожденных весов была найдена асимптотика с точностью до так называемой константы расхождения. Эта константа появилась в работе Ли [46] и имеет вид некоторого бесконечного произведения. Ее вычисление требует знания собственных функций ковариации. Используя подход, предложенный в [9], см. также близкие результаты в [38], Назаров и Пусев [10] вычислили константу расхождения для ряда взвешенных процессов Грина с известными собственными функциями. Для удобства читателя мы формулируем в 2 соответствующую теорему вместе с рядом вспомогательных результатов. Класс процессов, удовлетворяющих условиям теоремы 1 из $\S 2$, широк и включает, например, броуновское движение, броуновский мост, процесс Орнштейна-Уленбека и их многократно проинтегрированные аналоги.

В $\S 3$ мы показываем, как вычислить константу расхождения для ряда взвешенных гауссовских процессов, у которых собственные функиии ковариачии неизвестны, используя подход, тесно связанный с классическим методом ВКБ [37]. Это дает искомую точную асимптотику малых уклонений. Мы полагаем, что предложенный подход применим к значительно более широкому подклассу процессов Грина, чем процессы, рассмотренные в $\S 3$. 
В $\S 4$ результаты $\S 3$ используются для нахождения точной асимптотики малых уклонений во взвешенной $L_{2}$-норме для бесселевских процессов и мостов. В $\S 5$ получены аналогичные соотношения для броуновской экскурсии. Далее, в $\S 6$ и $\S 7$ мы исследуем такие же задачи для броуновского локального времени, для броуновского меандра и схожих процессов. В частности, рассматриваются интегральные функционалы от бесселевских процессов и броуновских локальных времен, попутно изучаются и супремумы рассматриваемых процессов. Результаты являются новыми даже в отсутствии веса, хотя их доказательства сравнительно просты и опираются на ряд известных тождеств по распределению между броуновскими функционалами.

Мы полагаем, что появление таблиц точных асимптотик для вероятностей малых уклонений - это лишь вопрос времени. Подобные таблицы будут похожими на таблицы интегралов, сумм и произведений и на таблицы распределений функционалов от броуновского движения.

2. Вспомогательные утверждения. Пусть $L-$ самосопряженный линейный дифференциальный оператор порядка $2 \ell$, определенный на пространстве $\mathscr{D}(L)$ функций, удовлетворяющих $2 \ell$ граничным условиям. Обозначим $W_{p}^{m}(0,1)$ банахово пространство $(m-1)$ раз непрерывно дифференцируемых функций $y$, у которых производная $y^{(m-1)}$ абсолютно непрерывна на $[0,1]$ и $y^{(m)} \in L_{p}(0,1)$. Следующая лемма доказана в [10, лемма 2.1].

Лемма 1. Рассмотрим функцию $\psi \in W_{\infty}^{\ell}(0,1)$ такую, что $\psi>0$ на $(0,1)$. Пусть $G(t, s)$ - функция Грина краевой задачи

$$
L v=\mu v \quad \text { на } \quad[0,1], \quad v \in \mathscr{D}(L) .
$$

Тогда функиия $\mathscr{G}(t, s)=\sqrt{\psi(t) \psi(s)} G(t, s)$ является функиией Грина краевой задачи

$$
\mathscr{L} v \equiv \psi^{-1 / 2} L\left(\psi^{-1 / 2} v\right)=\mu v \quad \text { на }[0,1], \quad v \in \mathscr{D}(\mathscr{L})
$$

где пространство $\mathscr{D}(\mathscr{L})$ состоит из функиий v, удовлетворяющих условию

$$
\psi^{-1 / 2} v \in \mathscr{D}(L) .
$$

3 а м е ч а н и е. Полагая $y=\psi^{-1 / 2} v$, можно переписать задачу (1)(2) следующим образом:

$$
L y=\mu \psi y \quad \text { на }[0,1], \quad y \in \mathscr{D}(L) .
$$

В той же работе [10] следующая теорема была доказана с помощью леммы 1 и теории, развитой в [53]. 
Теорема 1. Пусть ковариачионная функция $G_{X}(t, s)$ иентрированного гауссовского процесса $X(t), 0 \leqslant t \leqslant 1$, является функцией Грина самосопряженного дифференциального оператора $L$ порядка $2 \ell$

$$
\begin{gathered}
L v \equiv(-1)^{\ell} v^{(2 \ell)}+\left(p_{\ell-1} v^{(\ell-1)}\right)^{(\ell-1)}+\cdots+p_{0} v, \\
p_{m} \in L_{1}(0,1), \quad m=0, \ldots, \ell-2, \quad p_{\ell-1} \in L_{\infty}(0,1),
\end{gathered}
$$

с краевыми условиями

$$
\left.\begin{array}{l}
U_{j}^{0}(v) \equiv v^{\left(k_{j}\right)}(0)+\sum_{k<k_{j}} \alpha_{j k}^{0} v^{(k)}(0)=0, \\
U_{j}^{1}(v) \equiv v^{\left(k_{j}^{\prime}\right)}(1)+\sum_{k<k_{j}^{\prime}} \alpha_{j k}^{1} v^{(k)}(1)=0,
\end{array}\right\} \quad j=1, \ldots, \ell,
$$

где $\alpha_{j k}^{i}$ - некоторье постояннье,

$$
0 \leqslant k_{1}<\cdots<k_{\ell} \leqslant 2 \ell-1, \quad 0 \leqslant k_{1}^{\prime}<\cdots<k_{\ell}^{\prime} \leqslant 2 \ell-1 .
$$

Предположим, что $\varkappa \equiv \sum_{j=1}^{\ell}\left(k_{j}+k_{j}^{\prime}\right)<2 \ell^{2}$. Пусть $\psi \in W_{\infty}^{\ell}(0,1) u$ $\psi(x)>0, x \in[0,1]$. Тогда при $\varepsilon \rightarrow 0$

$$
\mathbf{P}\left(\|X\|_{\psi} \leqslant \varepsilon\right) \sim \mathscr{C} \varepsilon^{\gamma} \exp \left(-\frac{2 \ell-1}{2}\left(\frac{\vartheta_{\ell}}{2 \ell \sin (\pi /(2 \ell))}\right)^{2 \ell /(2 \ell-1)} \varepsilon^{-2 /(2 \ell-1)}\right),
$$

где

$$
\begin{gathered}
\gamma=-\ell+\frac{\varkappa+1}{2 \ell-1}, \quad \vartheta_{\ell}=\int_{0}^{1} \psi^{1 /(2 \ell)}(x) d x, \\
\mathscr{C}=C_{\text {dist }} \frac{(2 \pi)^{\ell / 2}\left(\pi / \vartheta_{\ell}\right)^{\ell \gamma}(\sin (\pi /(2 \ell)))^{(1+\gamma) / 2}}{(2 \ell-1)^{1 / 2}(\pi /(2 \ell))^{1+\gamma / 2} \Gamma^{\ell}(\ell-\varkappa /(2 \ell))} .
\end{gathered}
$$

$B(3)$ «онстанта расхождения» $C_{\text {dist }}$ задается формулой

$$
C_{\mathrm{dist}}=\prod_{n=1}^{\infty} \frac{\mu_{n}^{1 / 2}}{\left(\pi / \vartheta_{\ell} \cdot[n+\ell-1-\varkappa /(2 \ell)]\right)^{\ell}},
$$

где $\mu_{n}$ собственные числа краевой задачи

$$
L y=\mu \psi y, \quad U_{j}^{0}(y)=0, \quad U_{j}^{1}(y)=0, \quad j=1, \ldots, \ell .
$$

Далее нам понадобится лемма М. А. Лифшица (см. также [27], [12]), которая доказывается прямыми, хотя и трудоемкими вычислениями.

Лемма 2. Пусть $V_{1}, V_{2}>0-$ две независимьх случайных величинь (с.в.) с известными асимптотиками мальх уклонений. А именно, допустим, ито при $r \rightarrow 0$

$$
\begin{aligned}
& \mathbf{P}\left\{V_{1} \leqslant r\right\} \sim K_{1} r^{a_{1}} \exp \left(-D_{1}^{d+1} r^{-d}\right), \\
& \mathbf{P}\left\{V_{2} \leqslant r\right\} \sim K_{2} r^{a_{2}} \exp \left(-D_{2}^{d+1} r^{-d}\right),
\end{aligned}
$$


где $a_{1}, a_{2}$ - произвольные вещественные числа, а $K_{1}, K_{2}, D_{1}, D_{2}, d-$ произвольнье положительные числа. Тогда

$$
\mathbf{P}\left\{V_{1}+V_{2} \leqslant r\right\} \sim K r^{a} \exp \left(-D^{d+1} r^{-d}\right),
$$

где

$$
D=D_{1}+D_{2}, \quad a=a_{1}+a_{2}-\frac{d}{2}, \quad K=K_{1} K_{2} \sqrt{\frac{2 \pi d}{d+1}} \cdot \frac{D_{1}^{a_{1}+1 / 2} D_{2}^{a_{2}+1 / 2}}{D^{a+1 / 2}} .
$$

C помощью индукции по $n$ лемма 2 легко обобщается на случай произвольного числа независимых одинаково распределенных (н.о.р.) с.в. Справедлив следующий результат.

Лемма 3. Пусть $V_{1}, \ldots, V_{n}-$ н.о.р. положительные с.в. такие, чmo

$$
\mathbf{P}\left\{V_{i} \leqslant r\right\} \sim K r^{a} \exp \left(-D^{2} r^{-1}\right), \quad r \rightarrow 0,
$$

где а - вещественное число, а $K$ и $D$ - положительные постоянные. Тогда

$$
\mathbf{P}\left\{V_{1}+\cdots+V_{n} \leqslant r\right\} \sim \widetilde{K} r^{\tilde{a}} \exp \left(-\widetilde{D}^{2} r^{-1}\right), \quad r \rightarrow 0,
$$

гдe

$$
\widetilde{D}=n D, \quad \widetilde{a}=n a-\frac{n-1}{2}, \quad \widetilde{K}=\frac{K^{n} D^{n-1} \pi^{(n-1) / 2}}{n^{\widetilde{a}+1 / 2}} .
$$

3. Малые уклонения во взвешенной квадратичной норме. Обозначим

$$
\vartheta=\int_{0}^{1} \sqrt{\psi(t)} d t
$$

Следующая теорема дает точную асимптотику малых уклонений для броуновского моста $B$ во взвешенной $L_{2}$-норме для широкого класса весов.

Теорема 2. Пусть функиия $\psi$ определена на $[0,1]$, положительна и дважды непрерьвно дифферениируема. Тогда при $\varepsilon \rightarrow 0$

$$
\mathbf{P}\left\{\|B\|_{\psi} \leqslant \varepsilon\right\} \sim \frac{2 \sqrt{2} \psi^{1 / 8}(0) \psi^{1 / 8}(1)}{\sqrt{\pi \vartheta}} \exp \left(-\frac{\vartheta^{2}}{8} \varepsilon^{-2}\right) .
$$

Д о к а з а т е л ь с т в о. В соответствии с леммой 1 , коэффициенты $\lambda_{k}$ в разложении Кархунена-Лоэва имеют вид $\lambda_{k}=\mu_{k}^{-1}$, где $\mu_{k}$ собственные числа краевой задачи

$$
\left\{\begin{array}{l}
-y^{\prime \prime}=\mu \psi y \quad \text { на }[0,1] \\
y(0)=y(1)=0
\end{array}\right.
$$


Пусть $\varphi_{1}(t, \zeta)$ и $\varphi_{2}(t, \zeta)$ решения уравнения $-y^{\prime \prime}=\zeta^{2} \psi y$, удовлетворяющие начальным условиям

$$
\begin{aligned}
& \varphi_{1}(0, \zeta)=1, \quad \varphi_{1}^{\prime}(0, \zeta)=0 \\
& \varphi_{2}(0, \zeta)=0, \quad \varphi_{2}^{\prime}(0, \zeta)=1
\end{aligned}
$$

Такой выбор фундаментальной системы решений удобен. Он позволяет нам исследовать поведение решений по $\zeta$ как в окрестности нуля, так и на бесконечности.

Подставляя общее решение $y(t)=c_{1} \varphi_{1}(t, \zeta)+c_{2} \varphi_{2}(t, \zeta)$ в краевые условия, мы видим, что $\mu_{k}=x_{k}^{2}$, где $x_{1}<x_{2}<\cdots-$ положительные корни функции

$$
F(\zeta)=\operatorname{det}\left[\begin{array}{ll}
\varphi_{1}(0, \zeta) & \varphi_{2}(0, \zeta) \\
\varphi_{1}(1, \zeta) & \varphi_{2}(1, \zeta)
\end{array}\right]=\varphi_{2}(1, \zeta)
$$

В силу теоремы 1 , достаточно доказать, что

$$
C_{\text {dist }}^{2} \equiv \prod_{k=1}^{\infty} \frac{x_{k}^{2}}{(\pi k / \vartheta)^{2}}=\frac{\psi^{1 / 4}(0) \psi^{1 / 4}(1)}{\vartheta}
$$

Мы вычислим это бесконечное произведение с помощью теоремы Йенсена. Пусть $f(\zeta)$ - функция комплексного аргумента, которая аналитична при $|\zeta|<R$. Допустим, что $f(0) \neq 0$, и пусть $r_{1}, r_{2}, \ldots-$ модули нулей функции $f(\zeta)$ в круге $|\zeta|<R$, расположенные в неубывающем порядке. По теореме Йенсена (см., например, $[17, \S 3.6 .1])$ для $r_{k}<r<r_{k+1}$ имеем

$$
\ln \left(\frac{r^{k}|f(0)|}{r_{1} r_{2} \ldots r_{k}}\right)=\frac{1}{2 \pi} \int_{0}^{2 \pi} \ln \left|f\left(r e^{i \theta}\right)\right| d \theta .
$$

Поэтому для двух функций $f$ и $g$ с модулями нулей $r_{1}, r_{2}, \ldots$ и $s_{1}, s_{2}, \ldots$ соответственно, для всех $\max \left\{r_{k}, s_{k}\right\}<r<\min \left\{r_{k+1}, s_{k+1}\right\}$ получаем

$$
\ln \left(\frac{|f(0)| s_{1} s_{2} \ldots s_{k}}{|g(0)| r_{1} r_{2} \ldots r_{k}}\right)=\frac{1}{2 \pi} \int_{0}^{2 \pi} \ln \frac{\left|f\left(r e^{i \theta}\right)\right|}{\left|g\left(r e^{i \theta}\right)\right|} d \theta .
$$

Следовательно,

$$
\prod_{k=1}^{\infty} \frac{r_{k}}{s_{k}}=\left|\frac{f(0)}{g(0)}\right| \lim _{r \rightarrow \infty} \exp \left\{\frac{1}{2 \pi} \int_{0}^{2 \pi} \ln \frac{\left|g\left(r e^{i \theta}\right)\right|}{\left|f\left(r e^{i \theta}\right)\right|} d \theta\right\} .
$$

Для того, чтобы изучить асимптотику функции $F(\zeta)$ при $|\zeta| \rightarrow \infty$, мы используем так называемую ВКБ-аппроксимацию, которая давно используется в квантовой механике для анализа уравнения Шрёдингера, cM. [37]. 
В соответствии с $[22$, гл. $2, \S 3]$, уравнение $-y^{\prime \prime}=\zeta^{2} \psi y$ имеет решения вида

$$
\widetilde{\varphi}_{1,2}(t, \zeta)=\psi^{-1 / 4}(t) \exp \left( \pm i \zeta \int_{0}^{t} \sqrt{\psi(u)} d u\right)\left(1+\frac{\delta_{1,2}(t, \zeta)}{\zeta}\right)
$$

где функции $\delta_{1,2}(t, \zeta)$ удовлетворяют условию $\left|\delta_{1,2}(t, \zeta)\right| \leqslant C$ равномерно по $t \in[0,1]$ и $\zeta \in D_{r}^{-}=\{|\zeta| \geqslant r>0, \operatorname{Im}(\zeta) \leqslant 0\}$. Дифференцируя асимптотические выражения для функций $\widetilde{\varphi}_{1,2}(t, \zeta)$, получаем

$$
\widetilde{\varphi}_{1,2}^{\prime}(t, \zeta)= \pm i \zeta \psi^{1 / 4}(t) \exp \left( \pm i \zeta \int_{0}^{t} \sqrt{\psi(u)} d u\right)\left(1+\frac{\widetilde{\delta}_{1,2}(t, \zeta)}{\zeta}\right)
$$

где функции $\widetilde{\delta}_{1,2}(t, \zeta)$ также равномерно ограничены.

Заметим, что вронскиан решений $\widetilde{\varphi}_{1,2}(t, \zeta)$ не обращается в нуль, когда $|\zeta|$ растет. Поэтому для достаточно больших $|\zeta|$ функции $\widetilde{\varphi}_{1,2}(t, \zeta)$ линейно независимы, так что для больших $|\zeta|$ функции $\varphi_{1,2}(t, \zeta)$ могут быть представлены в виде линейных комбинаций функций $\widetilde{\varphi}_{1,2}(t, \zeta)$ :

$$
\begin{aligned}
& \varphi_{1}(t, \zeta)=c_{11}(\zeta) \widetilde{\varphi}_{1}(t, \zeta)+c_{12}(\zeta) \widetilde{\varphi}_{2}(t, \zeta) \\
& \varphi_{2}(t, \zeta)=c_{21}(\zeta) \widetilde{\varphi}_{1}(t, \zeta)+c_{22}(\zeta) \widetilde{\varphi}_{2}(t, \zeta)
\end{aligned}
$$

Благодаря условиям (5)-(6) мы имеем

$$
\begin{gathered}
c_{11}(\zeta)=\frac{\psi^{1 / 4}(0)}{2}+O\left(\zeta^{-1}\right), \quad c_{12}(\zeta)=\frac{\psi^{1 / 4}(0)}{2}+O\left(\zeta^{-1}\right), \\
c_{21}(\zeta)=\frac{1}{2 i \zeta \psi^{1 / 4}(0)}+O\left(\zeta^{-2}\right), \quad c_{22}(\zeta)=-\frac{1}{2 i \zeta \psi^{1 / 4}(0)}+O\left(\zeta^{-2}\right) .
\end{gathered}
$$

Значит, следующие соотношения справедливы при $|\zeta| \rightarrow \infty, \operatorname{Im}(\zeta) \leqslant 0$ :

$$
\begin{aligned}
\varphi_{1}(1, \zeta) & =\frac{\psi^{1 / 4}(0) \cos (\vartheta \zeta)}{\psi^{1 / 4}(1)}\left(1+O\left(\zeta^{-1}\right)\right), \\
\varphi_{1}^{\prime}(1, \zeta) & =-\psi^{1 / 4}(0) \psi^{1 / 4}(1) \zeta \sin (\vartheta \zeta)\left(1+O\left(\zeta^{-1}\right)\right), \\
\varphi_{2}(1, \zeta) & =\frac{\sin (\vartheta \zeta)}{\psi^{1 / 4}(0) \psi^{1 / 4}(1) \zeta}\left(1+O\left(\zeta^{-1}\right)\right), \\
\varphi_{2}^{\prime}(1, \zeta) & =\frac{\psi^{1 / 4}(1) \cos (\vartheta \zeta)}{\psi^{1 / 4}(0)}\left(1+O\left(\zeta^{-1}\right)\right) .
\end{aligned}
$$

Аналогично доказывается, что сходные соотношения справедливы при $|\zeta| \rightarrow \infty, \operatorname{Im}(\zeta) \geqslant 0$. Итак, мы получаем при $|\zeta| \rightarrow \infty$ :

$$
F(\zeta)=\varphi_{2}(1, \zeta)=\frac{\sin (\vartheta \zeta)}{\psi^{1 / 4}(0) \psi^{1 / 4}(1) \zeta}\left(1+O\left(\zeta^{-1}\right)\right) .
$$


Положим $\Psi(\zeta)=\sin (\vartheta \zeta) /(\vartheta \zeta)$. Для больших $k$ в круге $|\zeta|<$ $\pi / \vartheta(k+1 / 2)$ лежат ровно $2 k$ нулей $\pm \pi / \vartheta, \pm 2 \pi / \vartheta, \ldots, \pm k \pi / \vartheta$ функции $\Psi(\zeta)$ и ровно $2 k$ нулей $\pm x_{j}, j=1, \ldots, k$, функции $F(\zeta)$. Применяя теорему Йенсена к функциям $F(\zeta)$ и $\Psi(\zeta)$, получаем

$$
C_{\text {dist }}^{2}=\frac{\psi^{1 / 4}(0) \psi^{1 / 4}(1)|F(0)|}{\vartheta} .
$$

Из непрерывной зависимости решения дифференциального уравнения от параметра (см., например, [32, гл. $1, \S 7]$ ) следует, что $F(0)=$ $\lim _{\zeta \rightarrow 0} \varphi_{2}(1, \zeta)=\lim _{t \rightarrow 1} \varphi_{2}(t, 0)$. Ясно, что $\varphi_{2}(t, 0)=t$. Поэтому $F(0)=1$. Теорема 1 доказана.

3 а м е ч а н и е. Выбор $\psi \equiv 1$ и $\psi(t)=\exp (q t)$ в соотношении (4), приводит к известному результату о малых уклонениях броуновского моста и к формуле (3.16) из [9].

Далее вес $\psi$, удовлетворяющий условиям теоремы 2 , будем называть правильнылм.

В следующей теореме мы находим асимптотику малых уклонений для «удлиненного» броуновского моста $W_{(u)}(t) \equiv W(t)-u t W(1)$. Это центрированный гауссовский процесс с ковариацией $G_{W_{(u)}}(t, s)=$ $s \wedge t-\left(2 u-u^{2}\right) s t$, так что при $u \in(0,1]$ процесс $W_{(u)}$ совпадает по распределению с броуновским мостом из нуля в нуль длины $\left(2 u-u^{2}\right)^{-1}$ на интервале $[0,1]$. При $u=1$ этот процесс совпадает со стандартным броуновским мостом. При $u=0$ это стандартное броуновское движение. Ковариация $G_{W_{(u)}}$ является функцией Грина краевой задачи:

$$
\left\{\begin{array}{l}
-y^{\prime \prime}=\mu \psi y \quad \text { на }[0,1] \\
y(0)=\left(y^{\prime}+\tau y\right)(1)=0
\end{array}\right.
$$

где $\tau=(1-u)^{-2}-1$. С помощью тех же рассуждений, что и выше, мы можем найти точную асимптотику малых уклонений для процесса $W_{(u)}(t)$ в весовой норме.

Теорема 3. Рассмотрим прочесс $W_{(u)}(t)$ при $u<1$, и предположим, что $\psi-$ правильный вес на $[0,1]$. Тогда при $\varepsilon \rightarrow 0$

$$
\mathbf{P}\left\{\left\|W_{(u)}\right\|_{\psi} \leqslant \varepsilon\right\} \sim \frac{4 \psi^{1 / 8}(0)}{(1-u) \sqrt{\pi} \vartheta \psi^{1 / 8}(1)} \varepsilon \exp \left(-\frac{\vartheta^{2}}{8} \varepsilon^{-2}\right) .
$$

3 а м е ч а н и е. Для $u=0$ и $\psi \equiv 1$ соотношение (13) является классическим. Для $\psi(t)=\exp (q t)$ из формулы (13) вытекает формула (3.15) из [9]. Из теорем 2 и 3 следуют теоремы $3.1-3.4$ и теорема 4.1 в [10].

Две следующие теоремы описывают точную асимптотику малых уклонений для обычного стационарного процесса Орнштейна-Уленбека (для краткости ОУ), а также процесса ОУ, выходящего из нуля. Обозначим $U_{(\alpha)}(t)$ стационарный процесс ОУ, т.е. центрированный гауссовский процесс с ковариацией $G_{U_{(\alpha)}}(t, s)=e^{-\alpha|t-s|} /(2 \alpha)$. 
Теорема 4. Рассмотрим проиесс $U_{(\alpha)}(t), \alpha>0$, и пусть $\psi-n p a-$ вильньий вес на $[0,1]$. Тогда при $\varepsilon \rightarrow 0$

$$
\mathbf{P}\left\{\left\|U_{(\alpha)}\right\|_{\psi} \leqslant \varepsilon\right\} \sim \frac{8 \alpha^{1 / 2} e^{\alpha / 2}}{\pi^{1 / 2} \vartheta^{3 / 2} \psi^{1 / 8}(0) \psi^{1 / 8}(1)} \varepsilon^{2} \exp \left(-\frac{\vartheta^{2}}{8} \varepsilon^{-2}\right) .
$$

Д о к а з а т е л ь с т в о. По лемме 1 имеем $\lambda_{k}=\mu_{k}^{-1}$, где $\mu_{k}-$ собственные числа задачи

$$
\left\{\begin{array}{l}
y^{\prime \prime}+\left(\mu \psi-\alpha^{2}\right) y=0 \quad \text { на }[0,1], \\
\left(y^{\prime}-\alpha y\right)(0)=\left(y^{\prime}+\alpha y\right)(1)=0 .
\end{array}\right.
$$

Обозначим $\varphi_{1,2}(t, \zeta)$ решения уравнения $y^{\prime \prime}+\left(\zeta^{2} \psi-\alpha^{2}\right) y=0$, удовлетворяющие условиям (5)-(6). Подставляя общее решение $y(t)=$ $c_{1} \varphi_{1}(t, \zeta)+c_{2} \varphi_{2}(t, \zeta)$ в краевые условия, находим $\mu_{k}=x_{k}^{2}$, где $x_{1}<x_{2}<$ $\cdots$ - положительные нули функции

$$
\begin{aligned}
F(\zeta) & =\operatorname{det}\left[\begin{array}{ll}
\varphi_{1}^{\prime}(0, \zeta)-\alpha \varphi_{1}(0, \zeta) & \varphi_{2}^{\prime}(0, \zeta)-\alpha \varphi_{2}(0, \zeta) \\
\varphi_{1}^{\prime}(1, \zeta)+\alpha \varphi_{1}(1, \zeta) & \varphi_{2}^{\prime}(1, \zeta)+\alpha \varphi_{2}(1, \zeta)
\end{array}\right] \\
& =-\alpha \varphi_{2}^{\prime}(1, \zeta)-\alpha^{2} \varphi_{2}(1, \zeta)-\varphi_{1}^{\prime}(1, \zeta)-\alpha \varphi_{1}(1, \zeta)
\end{aligned}
$$

Действуя, как при доказательстве теоремы 4.3 в [10], получаем

$$
\mathbf{P}\left\{\left\|U_{(\alpha)}\right\|_{\psi} \leqslant \varepsilon\right\} \sim C_{\text {dist }} \frac{4 \sqrt{2}}{\sqrt{\pi} \vartheta^{2}} \varepsilon^{2} \exp \left(-\frac{\vartheta^{2}}{8} \varepsilon^{-2}\right),
$$

где

$$
C_{\mathrm{dist}}^{2}=\vartheta^{2} x_{1}^{2} \prod_{k=2}^{\infty} \frac{x_{k}^{2}}{(\pi(k-1) / \vartheta)^{2}} .
$$

Остается показать, что

$$
C_{\mathrm{dist}}^{2}=\frac{2 \alpha \vartheta e^{\alpha}}{\psi^{1 / 4}(0) \psi^{1 / 4}(1)}
$$

В соответствии с $[22$, гл. $2, \S 3]$, уравнение $y^{\prime \prime}+\left(\zeta^{2} \psi-\alpha^{2}\right) y=0$ имеет решения $\widetilde{\varphi}_{1,2}(t, \zeta)$, удовлетворяющие соотношениям $(7),(8)$. Как и в доказательстве теоремы 2 , можно показать, что соотношения (9)-(12) верны для $\varphi_{1,2}(1, \zeta)$ и $\varphi_{1,2}^{\prime}(1, \zeta)$. Поэтому при $|\zeta| \rightarrow \infty$

$$
F(\zeta)=\psi^{1 / 4}(0) \psi^{1 / 4}(1) \zeta \sin (\vartheta \zeta)\left(1+O\left(\zeta^{-1}\right)\right)
$$

Обозначим $\Psi(\zeta)=\left((\vartheta \zeta)^{2}-1\right)(\sin (\vartheta \zeta) / \zeta)$. Применяя теорему Йенсена к функциям $F(\zeta)$ and $\Psi(\zeta)$, получаем

$$
C_{\mathrm{dist}}^{2}=\frac{\vartheta|F(0)|}{\psi^{1 / 4}(0) \psi^{1 / 4}(1)} .
$$


По непрерывной зависимости решения от параметра, имеем

$$
F(0)=\lim _{t \rightarrow 1}\left(-\alpha \varphi_{2}^{\prime}(t, 0)-\alpha^{2} \varphi_{2}(t, 0)-\varphi_{1}^{\prime}(t, 0)-\alpha \varphi_{1}(t, 0)\right) .
$$

Прямыми вычислениями получаем

$$
\varphi_{1}(t, 0)=\operatorname{ch}(\alpha t), \quad \varphi_{2}(t, 0)=\frac{1}{\alpha} \operatorname{sh}(\alpha t) .
$$

Следовательно $|F(0)|=2 \alpha e^{\alpha}$. Теорема 4 доказана.

3 а м е ч а н и е. Выбор $\psi(t)=\exp (q t)$ в (14) дает утверждение теоремы 4.3 из [10]; кроме того, теорема 4 обобщает известные результаты из [9, предл. 2.1], [38, следствие 3], и [21, с.140], полученные для единичного веса.

Процесс ОУ, выходящий из нуля, $\stackrel{\circ}{U}_{(\alpha)}(t)-$ это центрированный гауссовский процесс с ковариацией $G_{U_{(\alpha)}}(t, s)=\left(e^{-\alpha|t-s|}-e^{-\alpha(t+s)}\right) /(2 \alpha)$. Соответствующая граничная задача имеет вид:

$$
\left\{\begin{array}{l}
y^{\prime \prime}+\left(\mu \psi-\alpha^{2}\right) y=0 \quad \text { на }[0,1] \\
y(0)=\left(y^{\prime}+\alpha y\right)(1)=0
\end{array}\right.
$$

Используя те же аргументы, что и в доказательстве предыдущей теоремы, получаем точную асимптотику малых уклонений для процесса $\mathrm{U}_{(\alpha)}(t)$.

Теорема 5. Пусть $\psi-$ правильный вес на $[0,1]$. Тогда при $\varepsilon \rightarrow 0$

$$
\mathbf{P}\left\{\left\|\stackrel{\circ}{U}_{(\alpha)}\right\|_{\psi} \leqslant \varepsilon\right\} \sim \frac{4 e^{\alpha / 2} \psi^{1 / 8}(0)}{\sqrt{\pi} \vartheta \psi^{1 / 8}(1)} \varepsilon \exp \left(-\frac{\vartheta^{2}}{8} \varepsilon^{-2}\right) .
$$

3 а м е ч а н и е. Теорема 5 обобщает следствие 3 из [38], полученное для единичного веса. Она обобщает также теорему 4.2 из [10], отвечающую выбору $\psi(t)=\exp (q t)$ в (15).

В следующей теореме вычисляется точная асимптотика малых уклонений для процесса Боголюбова $Y(t), t \in[0,1]$. Это центрированный гауссовский процесс с ковариацией

$$
\mathbf{E} Y(t) Y(s)=\frac{1}{2 \omega \operatorname{sh}(\omega / 2)} \operatorname{ch}\left(\omega|t-s|-\frac{\omega}{2}\right), \quad t, s \in[0,1], \quad \omega>0 .
$$

Из теории гауссовских процессов известно, что траектории процесса Боголюбова непрерывны почти наверное (п.н.) Из (16) мы получаем $\mathbf{E}(Y(1)-Y(0))^{2}=0$, так что $Y(0)=Y(1)$ п.н. Таким образом, почти все траектории процесса Боголюбова лежат в пространстве $C^{0}[0,1]$ непрерывных функций $x(t)$ на $[0,1]$, удовлетворяющих условию $x(0)=x(1)$. Различные свойства этого процесса рассматривались, например, в [13], [14] и $[20]$. 
Ковариация процесса Боголюбова является функцией Грина краевой задачи

$$
\left\{\begin{array}{l}
y^{\prime \prime}+\left(\mu \psi-\omega^{2}\right) y=0 \quad \text { на }[0,1] \\
y(0)-y(1)=y^{\prime}(0)-y^{\prime}(1)=0 .
\end{array}\right.
$$

Здесь краевые условия не разделены, однако с помощью [54] можно доказать результат, аналогичный теореме 1 , и в случае неразделенных краевых условий.

Пусев (см. [12]) нашел точную асимптотику малых уклонений для этого процесса при единичном и экспоненциальном весе, а также исследовал многократно проинтегрированный процесс. Мы сформулируем сейчас взвешенный вариант его результата, доказательство которого вполне аналогично предыдущим теоремам.

Теорема 6. Рассмотрим прочесс Боголюбова $Y(t), t \in[0,1], u$ пусть $\psi-$ правильныи вес на $[0,1]$. Тогда при $\varepsilon \rightarrow 0$

$$
\mathbf{P}\left\{\|Y\|_{\psi} \leqslant \varepsilon\right\} \sim \frac{8 \operatorname{sh}(\omega / 2) \psi^{1 / 8}(0) \psi^{1 / 8}(1)}{\vartheta \pi^{1 / 2}\left(\psi^{1 / 2}(0)+\psi^{1 / 2}(1)\right)^{1 / 2}} \varepsilon \exp \left(-\frac{\vartheta^{2}}{8} \varepsilon^{-2}\right) .
$$

3 а м е ч а н и е. Подстановка $\psi \equiv 1$ и $\psi(t)=\exp (q t)$ в соотношение (17) приводит к утверждениям теоремы 1 и теоремы 2 из [12].

4. Малые уклонения бесселевских процессов. Переходим к изучению малых уклонений функционалов от некоторых негауссовских процессов, связанных с броуновским движением. Для негауссовских процессов использование случайных рядов типа Кархунена-Лоэва проблематично. Задача упрощается, если процесс выражается через простые гауссовские процессы.

Начнем с бесселевского процесса $\mathrm{Bes}^{\delta}$ размерности $\delta>0$, что соответствует индексу $\nu=\delta / 2-1 \in(-1, \infty)$. Определение бесселевских процессов и основные факты из их теории содержатся, например, в [4] и [58]. Для целых размерностей $n$ процесс $\mathrm{Bes}^{n}$ можно считать радиальной частью $n$-мерного броуновского движения с независимыми компонентами, в случае бесселевского моста $\mathrm{Bes}_{0}^{n}$ компонентами являются независимые броуновские мосты.

Асимптотика малых уклонений для многих функционалов от бесселевских процессов и мостов выводится из их точных распределений, собранных в [4]. Из формулы (4.1.9.4 (1)) в [4] следует, что для любой размерности $\delta \geqslant 2$ или любого индекса $\nu \geqslant 0$ при $r \rightarrow 0$

$\mathbf{P}\left\{\left\|\operatorname{Bes}^{\delta}\right\| \leqslant r\right\} \sim \frac{2^{\nu+3 / 2}}{(\nu+1) \sqrt{\pi}} r \exp \left(-\frac{(\nu+1)^{2}}{2 r^{2}}\right)=\frac{2^{(\delta+3) / 2}}{\delta \sqrt{\pi}} r \exp \left(-\frac{\delta^{2}}{8 r^{2}}\right)$. 
Малые уклонения бесселевских процессов и мостов в нормах $L_{p}$ изучались в [19]. Формула (18) следует из результатов работы [19] при $p=2$.

Точные распределения квадратичных норм для бесселевских мостов были найдены Кифером (см. [44]) в связи с задачами непараметрической статистики. По формуле Кифера для любого натурального $k$ и любого $a>0$

$$
\begin{aligned}
\mathbf{P}\left\{\left\|\operatorname{Bes}_{0}^{k}\right\|^{2} \leqslant a\right\}=\frac{2^{(k+1) / 2}}{\sqrt{\pi} a^{k / 4}} \sum_{j=0}^{\infty} & \frac{\Gamma(j+k / 2)}{j ! \Gamma(k / 2)} \exp \left(-\frac{(j+k / 4)^{2}}{a}\right) \\
& \times D_{(k-2) / 2}\left(\frac{2 j+k / 2}{\sqrt{a}}\right)
\end{aligned}
$$

где $D_{p}$ - функции параболического цилиндра.

Ясно, что при фиксированном индексе $p$ главную роль в асимптотике при $a \rightarrow 0$ играет первый член. При этом в силу $[2, \S 8.4]$ мы знаем, что при $z \rightarrow \infty$

$$
D_{p}(z) \sim z^{p} \exp \left(-z^{2} / 4\right), \quad z \rightarrow \infty .
$$

Поэтому при любом натуральном $k$ и при $\varepsilon \rightarrow 0$

$$
\mathbf{P}\left\{\left\|\operatorname{Bes}_{0}^{k}\right\| \leqslant \varepsilon\right\} \sim \frac{2 \sqrt{2}}{\sqrt{\pi}} k^{(k-2) / 2} \varepsilon^{-(k-1)} \exp \left(-\frac{k^{2}}{8 \varepsilon^{2}}\right) .
$$

В случае произвольной размерности $\delta>1$, формулы (19) и (20) остаются верными. Достаточно скомбинировать формулы (4.1.0.6) и (4.1.9.8) из справочника [4].

Теперь мы затронем вопрос о малых уклонениях супремума бесселевских процессов и мостов. Обозначим $\mu(Z)$ супремум случайного процесса $Z$ на $[0,1]$. Распределение супремума бесселевского моста целой размерности было найдено независимо Гихманом [5] и Кифером [44]; позже Питмен и Йор [57] доказали его справедливость для любой положительной размерности $\delta>0$ или индекса $\nu=(\delta-2) / 2$.

Пусть $0<j_{\nu, 1}<j_{\nu, 2}<\cdots$ - последовательность положительных нулей функции Бесселя $J_{\nu}$. Из формулы Гихмана-Кифера-ПитменаЙора следует, что при $\nu>-1$ (т.е. при $\delta>0$ ) и при любом $r \geqslant 0$ верна формула

$$
\mathbf{P}\left\{\mu\left(\operatorname{Bes}_{0}^{\delta}\right) \leqslant r\right\}=\left(2^{\nu-1} \Gamma(\nu+1) r^{\nu}\right)^{-1} \sum_{n=1}^{\infty} \frac{j_{\nu, n}^{2 \nu}}{J_{\nu+1}^{2}\left(j_{\nu, n}\right)} \exp \left(-\frac{j_{\nu, n}^{2}}{2 r^{2}}\right) .
$$

Очевидно, что главный вклад при $r \rightarrow 0$ вносит первый член. Поэтому точная асимптотика малых уклонений для супремума бесселевского моста при $\nu>-1$ принимает вид:

$$
\mathbf{P}\left\{\mu\left(\operatorname{Bes}_{0}^{\delta}\right) \leqslant r\right\} \sim\left(2^{\nu-1} \Gamma(\nu+1) r^{2 \nu+2}\right)^{-1} \frac{j_{\nu, 1}^{2 \nu}}{J_{\nu+1}^{2}\left(j_{\nu, 1}\right)} \exp \left(-\frac{j_{\nu, 1}^{2}}{2 r^{2}}\right) .
$$


Аналогичная формула для бесселевского процесса выглядит несколько иначе. Из справочника [4, формула 4.1.1.4] (см. также [57]) вытекает что при $\nu>-1$

$$
\mathbf{P}\left\{\mu\left(\operatorname{Bes}^{\delta}\right) \leqslant r\right\}=\left(2^{\nu-1} \Gamma(\nu+1)\right)^{-1} \sum_{n=1}^{\infty} \frac{j_{\nu, n}^{\nu-1}}{J_{\nu+1}\left(j_{\nu, n}\right)} \exp \left(-\frac{j_{\nu, n}^{2}}{2 r^{2}}\right) .
$$

Эта формула для целых размерностей была впервые получена в знаменитой работе [31]. Снова основной вклад при $r \rightarrow 0$ принадлежит первому члену. Отсюда следует формула: при $r \rightarrow 0$

$$
\mathbf{P}\left\{\mu\left(\operatorname{Bes}^{\delta}\right) \leqslant r\right\} \sim\left(2^{\nu-1} \Gamma(\nu+1)\right)^{-1} \frac{j_{\nu, 1}^{\nu-1}}{J_{\nu+1}\left(j_{\nu, 1}\right)} \exp \left(-\frac{j_{\nu, 1}^{2}}{2 r^{2}}\right) .
$$

Частные случаи формул (21) и (22) для малых натуральных размерностей обсуждаются в обзоре Фаталова [18].

Хорошо известно, что степени бесселевских процессов принадлежат к тому же семейству процессов с точностью до подходящей замены времени $[58$, гл. ХI]. Это приводит к ряду тождеств по распределению между интегралами от различных степеней бесселевских процессов, см. [58, гл. XI], [29], [30]. Возьмем в качестве примера типичное тождество [58, следствие 1.12, гл. XI], верное при целых размерностях $d>1$ :

$$
4\left(\int_{0}^{1}\left(\operatorname{Bes}^{d}(s)\right)^{-1} d s\right)^{-2} \stackrel{\text { law }}{=} \int_{0}^{1}\left(\operatorname{Bes}^{2 d-2}(s)\right)^{2} d s=\left\|\operatorname{Bes}^{2 d-2}\right\|^{2} .
$$

По этому тождеству, точная асимптотика малых уклонений (18), которая справедлива для правой части, справедлива и для левой части. Другой пример дает тождество из [30, табл. 2]:

$$
\left\|\operatorname{Bes}_{0}^{4}\right\|^{2}=\int_{0}^{1}\left(\operatorname{Bes}_{0}^{4}(s)\right)^{2} d s \stackrel{\text { law }}{=}\left(\frac{1}{\pi} \int_{0}^{1}\left(\operatorname{Bes}_{0}^{3}(s)\right)^{-1} d s\right)^{2} .
$$

Пользуясь (20), мы получаем новое асимптотическое соотношение: при $r \rightarrow 0$

$$
\mathbf{P}\left\{\int_{0}^{1}\left(\operatorname{Bes}_{0}^{3}(s)\right)^{-1} d s \leqslant r\right\} \sim \frac{8 \pi^{2} \sqrt{2 \pi}}{r^{3}} \exp \left(-\frac{2 \pi^{2}}{r^{2}}\right) .
$$

Применяя лемму 3 к квадратам взвешенных норм броуновских движений и используя теорему 3 , мы получаем точную асимптотику малых уклонений для взвешенной нормы бесселевского процесса целой размерности.

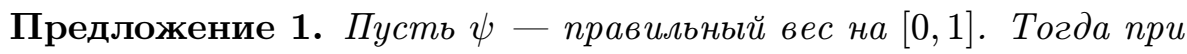
$\varepsilon \rightarrow 0$

$$
\mathbf{P}\left\{\left\|\operatorname{Bes}^{k}\right\|_{\psi} \leqslant \varepsilon\right\} \sim \frac{2^{(k+3) / 2} \psi^{k / 8}(0)}{\sqrt{\pi} k \vartheta \psi^{k / 8}(1)} \varepsilon \exp \left(-\frac{k^{2} \vartheta^{2}}{8} \varepsilon^{-2}\right) .
$$


Аналогично устанавливается формула для бесселевского моста целой размерности.

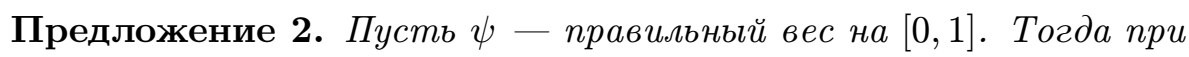
$\varepsilon \rightarrow 0$

$\mathbf{P}\left\{\left\|\operatorname{Bes}_{0}^{k}\right\|_{\psi} \leqslant \varepsilon\right\} \sim \frac{2 \sqrt{2}}{\sqrt{\pi}}(k \vartheta)^{(k-2) / 2} \psi^{k / 8}(0) \psi^{k / 8}(1) \varepsilon^{-(k-1)} \exp \left(-\frac{k^{2} \vartheta^{2}}{8} \varepsilon^{-2}\right)$.

Для дальнейшего использования опишем асимптотику малых уклонений для суммы норм $\left\|\mathrm{Bes}^{k}\right\|^{2}+\left\|\mathrm{Bes}_{0}^{m}\right\|^{2}$ в случае целых $k, m \geqslant 1$.

Лемма 4. Пусть $\mathrm{Bes}^{k}$ - бесселевский прочесс челой размерности $k \geqslant 1, a \operatorname{Bes}_{0}^{m}$ - независимый от него бесселевский мост иелой размерности $m \geqslant 1$. Тогда при $\varepsilon \rightarrow 0$

$$
\mathbf{P}\left\{\left\|\operatorname{Bes}^{k}\right\|^{2}+\left\|\operatorname{Bes}_{0}^{m}\right\|^{2} \leqslant \varepsilon^{2}\right\} \sim \frac{2^{(k+3) / 2} m^{m-1}}{\sqrt{\pi}(k+m)^{m / 2}} \varepsilon^{m-1} \exp \left(-\frac{(m+k)^{2}}{8 \varepsilon^{2}}\right) .
$$

Д о к а з а т е л ь с т в о. Доказательство заключается в «склеивании» двух асимптотик (18) и (20) с помощью леммы 2.

Другой пример использования леммы 3 таков. Рассмотрим несуммируемый вес $\omega(t)=[t(1-t)]^{-1}, 0 \leqslant t \leqslant 1$, называемый весом Андерсона-Дарлинга (см. [26]). Квадрат нормы броуновского моста с весом Андерсона-Дарлинга в обозначениях [51]

$$
A^{1,1}:=\int_{0}^{1} B^{2}(t) \omega(t) d t
$$

играет важную роль в теории непараметрических критериев согласия. В работе [9] было доказано, что при $r \rightarrow 0$

$$
\mathbf{P}\left\{A^{1,1} \leqslant r\right\} \sim \frac{2}{\sqrt{r}} \exp \left(-\frac{\pi^{2}}{8 r}\right) .
$$

Рассмотрим, следуя статье [51], для натуральных $n$ функционал

$$
A^{n, 1}:=\int_{0}^{1} \frac{\left(\operatorname{Bes}_{0}^{n}(t)\right)^{2}}{t(1-t)} d t
$$

Ясно, что $A^{n, 1}-$ сумма $n$ независимых копий величины $A^{1,1}$. Поэтому по лемме 3 и (25) мы получаем при $r \rightarrow 0$

$$
\mathbf{P}\left\{A^{n, 1} \leqslant r\right\} \sim 2^{(3-n) / 2}(n \pi \sqrt{\pi})^{n-1} r^{-n+1 / 2} \exp \left(-\frac{n^{2} \pi^{2}}{8 r}\right) .
$$

Обозначим теперь $T_{1}(3)$ и $T_{1}(4)$ моменты первого достижения уровня 1 бесселевскими процессами $\mathrm{Bes}^{3}$ и $\mathrm{Bes}^{4}$. Следующие два тождества по распределению доказаны в [51, с. 174]:

$$
A^{2,1} \stackrel{\text { law }}{=} \int_{0}^{T_{1}(3)} \frac{d s}{\operatorname{Bes}^{3}(s)\left(1-\operatorname{Bes}^{3}(s)\right)} \stackrel{\text { law }}{=} 4 \int_{0}^{T_{1}(4)} \frac{d u}{1-\left(\operatorname{Bes}^{4}(u)\right)^{2}} .
$$


Использование соотношения (26) при $n=2$ дает нам две новых точных асимптотики малых уклонений для бесселевских процессов при $r \rightarrow 0$ :

$$
\begin{aligned}
\mathbf{P}\left\{\int_{0}^{T_{1}(3)}\left(\operatorname{Bes}^{3}(s)\left(1-\operatorname{Bes}^{3}(s)\right)\right)^{-1} d s \leqslant r\right\} & \sim 2 \pi \sqrt{2 \pi} r^{-3 / 2} e^{-\pi^{2} / 2 r}, \\
\mathbf{P}\left\{\int_{0}^{T_{1}(4)}\left(1-\left(\operatorname{Bes}^{4}(u)\right)^{2}\right)^{-1} d u \leqslant r\right\} & \sim \frac{1}{4} \pi \sqrt{2 \pi} r^{-3 / 2} e^{-\pi^{2} / 8 r} .
\end{aligned}
$$

В заключение рассмотрим замечательный результат Алили (см. [24], [30, формула 4.33]), устанавливающий следующее тождество по распределению при любом $\sigma \neq 0$ :

$$
\frac{\sigma^{2}}{\pi^{2}}\left[\left(\int_{0}^{1} \operatorname{cth}\left(\sigma \operatorname{Bes}_{0}^{3}(u)\right) d u\right)^{2}-1\right] \stackrel{\text { law }}{=}\left\|\operatorname{Bes}_{0}^{4}\right\|^{2} .
$$

В силу (23) это может быть записано в форме

$$
\sigma^{2}\left[\left(\int_{0}^{1} \operatorname{cth}\left(\sigma \operatorname{Bes}_{0}^{3}(u)\right) d u\right)^{2}-1\right] \stackrel{\operatorname{law}}{=}\left(\int_{0}^{1}\left(\operatorname{Bes}_{0}^{3}(s)\right)^{-1} d s\right)^{2} .
$$

Используя (20), мы можем получить точную асимптотику малых уклонений для функционала в левой части (27) при любом $\sigma$. Как показано в [25] (см. также [30, формула 4.34]) при $\sigma \rightarrow \infty$ справедливо следующее тождество по распределению:

$$
\int_{0}^{\infty}\left(\exp \left(\operatorname{Bes}^{3}(t)\right)-1\right)^{-1} d t \stackrel{\text { law }}{=} \frac{\pi^{2}}{4}\left\|\operatorname{Bes}_{0}^{2}\right\|^{2} .
$$

Этот результат влечет за собой еще одну точную асимптотику малых уклонений, на этот раз для экспоненциального функционала от бесселевского процесса.

Предложение 3. При $r \rightarrow 0$ справедливо соотношение

$$
\mathbf{P}\left\{\int_{0}^{\infty}\left(\exp \left(\operatorname{Bes}^{3}(t)\right)-1\right)^{-1} d t \leqslant r\right\} \sim \sqrt{\frac{2 \pi}{r}} \exp \left(-\frac{\pi^{2}}{8 r}\right) .
$$

5. Малые уклонения для броуновской экскурсии. Обозначим $\mathfrak{e}(t), 0 \leqslant t \leqslant 1$, броуновскую экскурсию. Строгое определение этого процесса можно найти в [4], [59] или [58]. На неформальном уровне можно представлять себе его как броуновский мост из нуля в нуль, принимающий положительные значения внутри $[0,1]$, или как броуновское движение, выходящее из нуля, положительное внутри $[0,1]$ и впервые обращающееся в нуль в момент времени 1.

Для наших целей ключевую роль играет связь между броуновской экскурсией и бесселевскими мостами. Эта связь известна давно и была 
описана сначала Леви (см. [45]), затем Ито и Маккином (см. [41, § 2.9]) и Уильямсом (см. [61]). Позже броуновская экскурсия и связанные с нею процессы изучались в множестве работ, см., например, [58] и [52]. Однако, насколько нам известно, точная асимптотика малых уклонений этих процессов в $L_{2}$-норме была неизвестна.

Теорема 7. Пусть $\psi-$ правильный вес на $[0,1]$. Тогда при $\varepsilon \rightarrow 0$

$$
\mathbf{P}\left\{\|\mathfrak{e}\|_{\psi} \leqslant \varepsilon\right\} \sim \frac{2 \sqrt{6 \vartheta} \psi^{3 / 8}(0) \psi^{3 / 8}(1)}{\sqrt{\pi}} \varepsilon^{-2} \exp \left(-\frac{9 \vartheta^{2}}{8} \varepsilon^{-2}\right) .
$$

Д о к а з а т е л ь с т в о. Хорошо известно (см. [61], [59]) следующее тождество по распределению, иногда называемое тождеством ЛевиУильямса (см. [30, с. 454]):

$$
\left\{\mathfrak{e}^{2}(t), 0 \leqslant t \leqslant 1\right\} \stackrel{\text { law }}{=}\left\{B_{1}^{2}(t)+B_{2}^{2}(t)+B_{3}^{2}(t), 0 \leqslant t \leqslant 1\right\},
$$

где $B_{1}(t), B_{2}(t), B_{3}(t), 0 \leqslant t \leqslant 1$, - три независимых броуновских моста. Умножая обе части $(28)$ на вес $\psi$ и интегрируя, получаем

$$
\|\mathfrak{e}\|_{\psi}^{2} \stackrel{\text { law }}{=}\left\|B_{1}\right\|_{\psi}^{2}+\left\|B_{2}\right\|_{\psi}^{2}+\left\|B_{3}\right\|_{\psi}^{2} \stackrel{\text { law }}{=}\left\|\operatorname{Bes}_{0}^{3}\right\|_{\psi}^{2} .
$$

Применение предложения 2 при $k=3$ завершает доказательство.

При выборе в теореме 7 единичного веса получаем точную асимптотику малых уклонений для собственно броуновской экскурсии:

$$
\mathbf{P}\{\|\mathfrak{e}\| \leqslant \varepsilon\} \sim \frac{2 \sqrt{6}}{\sqrt{\pi}} \varepsilon^{-2} \exp \left(-\frac{9}{8} \varepsilon^{-2}\right), \quad \varepsilon \rightarrow 0 .
$$

В качестве еще одного примера рассмотрим вес $\chi(t)=(1+t)^{-4}$. Применяя теорему 2 , легко получаем, что при $\varepsilon \rightarrow 0$

$$
\mathbf{P}\left\{\|B\|_{\chi} \leqslant \varepsilon\right\} \sim \frac{2 \sqrt{2}}{\sqrt{\pi}} \exp \left(-\frac{1}{32} \varepsilon^{-2}\right)
$$

(этот результат также вытекает из теоремы 4 работы [10] при $a=1$ ).

Между прочим, это соотношение исправляет ошибочную формулу (13) статьи [40]. Рассматривая броуновскую экскурсию с весом $\chi(t)$, получаем из теоремы 7 , что

$$
\mathbf{P}\left\{\|\mathfrak{e}\|_{\chi} \leqslant \varepsilon\right\} \sim \sqrt{\frac{3}{2 \pi}} \varepsilon^{-2} \exp \left(-\frac{9}{32} \varepsilon^{-2}\right) .
$$

Как и при доказательстве теоремы 7, используя теоремы 3.1 и 3.3 из [9], а также пример с) из [11], мы можем установить точные асимптотики малых уклонений для броуновской экскурсии с различными «вырождающимися» весами. 
Предложение 4. Следующие соотношения справедливы при $\varepsilon \rightarrow 0$ :

$$
\begin{aligned}
& \mathbf{P}\left\{\int_{0}^{1} \frac{\mathfrak{e}^{2}(t)}{t(1-t)} d t \leqslant \varepsilon^{2}\right\} \sim \frac{9 \pi^{3}}{\varepsilon^{5}} \exp \left(-\frac{9 \pi^{2}}{8 \varepsilon^{2}}\right), \\
& \mathbf{P}\left\{\int_{0}^{1} \frac{\mathfrak{e}^{2}(t)}{t(2-t)} d t \leqslant \varepsilon^{2}\right\} \sim \frac{3^{7 / 8} \pi^{3 / 2}}{2^{5 / 4}} \varepsilon^{-11 / 4} \exp \left(-\frac{9 \pi^{2}}{32 \varepsilon^{2}}\right) .
\end{aligned}
$$

Пусть $\rho>-2$. Тогда при $\varepsilon \rightarrow 0$

$$
\begin{aligned}
\mathbf{P}\left\{\int_{0}^{1} t^{\rho} \mathfrak{e}^{2}(t) d t \leqslant \varepsilon^{2}\right\} \sim & \frac{4 \pi^{1 / 4}}{3^{(\rho-4) /(4(\rho+2))} \Gamma^{3 / 2}((\rho+3) /(\rho+2))} \\
& \times((\rho+2) \varepsilon)^{-(\rho+8) /(2(\rho+2))} \exp \left(-\frac{9}{2}((\rho+2) \varepsilon)^{-2}\right) .
\end{aligned}
$$

Известно следующее тождество по распределению для функционала типа Ватсона от броуновской экскурсии (см. [30, формула (4.20)]):

$$
\text { Wat }(\mathfrak{e}):=\int_{0}^{1}\left(\mathfrak{e}(t)-\int_{0}^{1} \mathfrak{e}(x) d x\right)^{2} d t \stackrel{\text { law }}{=} \frac{1}{4}\left\|\operatorname{Bes}_{0}^{2}\right\|^{2} .
$$

Применяя формулу (20) при $k=2$, получаем новую точную асимптотику при $r \rightarrow 0$ :

$$
\mathbf{P}\{\operatorname{Wat}(\mathfrak{e}) \leqslant r\} \sim \sqrt{\frac{2}{\pi r}} \exp \left(-\frac{1}{8 r}\right) .
$$

6. О малых уклонениях броуновского локального времени. Пусть $L_{t}^{x}(B)$ непрерывное по совокупности переменных локальное время броуновского моста $B$ в точке $x \in \mathbf{R}$ до времени $t \in[0,1]$. В силу следствия 2.2 работы [33], для натуральных $m$ справедливо тождество по распределению

$$
\int_{-\infty}^{\infty}\left(L_{1}^{x}(B)\right)^{m} d x \stackrel{\text { law }}{=} 2^{m-1} \int_{0}^{1}(\mathfrak{e}(t))^{m-1} d t
$$

Сначала рассмотрим случай $m=3$. Точная асимптотика малых уклонений функционала $\|\mathfrak{e}\|^{2}$ в правой части (30) известна из теоремы 7. Отсюда следует подобная асимптотика для интегрального функционала $\int_{-\infty}^{\infty}\left(L_{1}^{x}(B)\right)^{3} d x$.

Предложение 5. При $\varepsilon \rightarrow 0$ справедливо соотношение:

$$
\mathbf{P}\left\{\int_{-\infty}^{\infty}\left(L_{1}^{x}(B)\right)^{3} d x \leqslant \varepsilon\right\} \sim \frac{8 \sqrt{6}}{\sqrt{\pi}} \varepsilon^{-1} \exp \left(-\frac{9}{2} \varepsilon^{-1}\right) .
$$

Это предложение уточняет результат из [33], где была найдена лишь логарифмическая асимптотика. 
Переходим к случаю $m=2$. Справедливо тождество по распределению

$$
\int_{-\infty}^{\infty}\left(L_{1}^{x}(B)\right)^{2} d x \stackrel{\text { law }}{=} 2 \int_{0}^{1} \mathfrak{e}(t) d t .
$$

Интеграл в правой части интерпретируется как площадь под броуновской экскурсией. Он интенсивно изучался (см. [42]). Его распределение можно описать так.

Пусть $A i(x)$ - стандартная функция Эйри (см. [15, гл. 10]). Все нули функции Эйри отрицательны. Обозначим их $-a_{j}, j \geqslant 1$, и пусть $a_{1}$ - абсолютная величина первого нуля, $a_{1} \approx 2.3381$. При $r \rightarrow 0+$ справедливо асимптотическое разложение (см. [42, § 15]):

$\mathbf{P}\left\{\int_{0}^{1} \mathfrak{e}(t) d t \leqslant r\right\} \sim \exp \left(-\frac{2 a_{1}^{3}}{27 r^{2}}\right)\left(\frac{2}{3} a_{1}^{3 / 2} r^{-2}+\frac{1}{4} a_{1}^{-3 / 2}-\frac{105}{64} a_{1}^{-9 / 2} r^{2}+\cdots\right)$,

причем главный вклад вносит первый член. Отсюда следует точная асимптотика малых уклонений для функционала $\int_{-\infty}^{\infty}\left(L_{1}^{x}(B)\right)^{2} d x$.

Предложение 6. При $\varepsilon \rightarrow 0$

$$
\mathbf{P}\left\{\int_{-\infty}^{\infty}\left(L_{1}^{x}(B)\right)^{2} d x \leqslant \varepsilon\right\} \sim \frac{8}{3} a_{1}^{3 / 2} \varepsilon^{-2} \exp \left(-\frac{8 a_{1}^{3}}{27 \varepsilon^{2}}\right) .
$$

Менее точный вариант этого результата на логарифмическом уровне был получен в [33, теорема 3.1]. Точная асимптотика, повидимому, выписана нами впервые.

Результаты о малых уклонениях броуновского локального времени вообще редки. Мы пополним их следствием из первой теоремы РэяНайта (см. [58, гл. XI], [52, гл. 3]). Пусть $T_{1}=\inf \{t: W(t)=1\}-$ первый момент достижения броуновским движением уровня 1 . Для $x \in[0,1]$ рассмотрим процесс локального времени по $x$ вплоть до момента $T_{1}$ :

$$
Z(x)=L_{T_{1}}^{x}(W), \quad 0 \leqslant x \leqslant 1 .
$$

Первая теорема Рэя-Найта утверждает, что на интервале $[0,1]$ этот процесс совпадает по распределению с квадратом процесса $\mathrm{Bes}^{2}$. Поэтому мы получаем при $\varepsilon \rightarrow 0$

$$
\mathbf{P}\left\{\int_{0}^{1} L_{T_{1}}^{x}(W) d x \leqslant \varepsilon^{2}\right\} \sim \frac{2 \sqrt{2}}{\sqrt{\pi}} \varepsilon \exp \left(-\frac{1}{2 \varepsilon^{2}}\right) .
$$

Обобщение этого результата с помощью предложения 1 для взвешенной квадратичной нормы выглядит так.

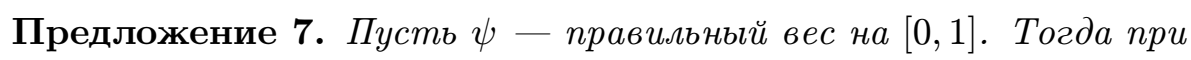
$\varepsilon \rightarrow 0$

$$
\mathbf{P}\left\{\int_{0}^{1} L_{T_{1}}^{x}(W) \psi(x) d x \leqslant \varepsilon^{2}\right\} \sim \frac{2 \sqrt{2} \psi^{1 / 4}(0)}{\sqrt{\pi} \vartheta \psi^{1 / 4}(1)} \varepsilon \exp \left(-\frac{\vartheta^{2}}{2} \varepsilon^{-2}\right) .
$$


Используя связь между процессом $L_{T_{1}}^{x}(W)$ и $\mathrm{Bes}^{2}$, можно найти точную асимптотику малых уклонений для $L_{p}$-нормы процесса $L_{T_{1}}^{x}(W)$. Для любого $p>0$ имеем

$$
\mathbf{P}\left\{\int_{0}^{1}\left(L_{T_{1}}^{x}(W)\right)^{p} d x \leqslant \varepsilon^{p}\right\}=\mathbf{P}\left\{\int_{0}^{1}\left(\operatorname{Bes}^{2}(t)\right)^{2 p} d t \leqslant \varepsilon^{p}\right\},
$$

где асимптотика правой части, пусть в неявной форме, может быть получена из [19].

Со случайным процессом $X(t)$, выходящим из нуля, можно связать так называемый супремум-процесс $S(t)=\sup _{0 \leqslant s \leqslant t} X(s)$. Для броуновского движения $W$ классический результат Леви (см. [45]) утверждает, что процесс $S(t)-W(t), t \geqslant 0$, совпадает по распределению с одномерным бесселевским процессом. Применяя теорему 3 , получаем следующее предложение.

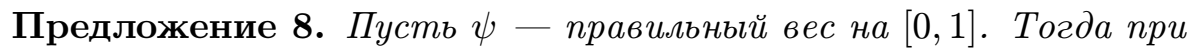
$\varepsilon \rightarrow 0$

$$
\mathbf{P}\left\{\|S-W\|_{\psi} \leqslant \varepsilon\right\} \sim \frac{4 \psi^{1 / 8}(0)}{\sqrt{\pi} \vartheta \psi^{1 / 8}(1)} \varepsilon \exp \left(-\frac{\vartheta^{2}}{8} \varepsilon^{-2}\right) .
$$

Другой замечательный результат принадлежит Питмену (см. [55], $[58$, гл. 6$])$, доказавшему совпадение по распределению процесса $2 S-W$ и процесса $\mathrm{Bes}^{3}$. Тождество Питмена влечет за собой равенство

$$
\mathbf{P}\left\{\|2 S-W\|_{\psi} \leqslant \varepsilon\right\}=\mathbf{P}\left\{\left\|\operatorname{Bes}^{3}\right\|_{\psi} \leqslant \varepsilon\right\} .
$$

Отсюда с помощью леммы 3 можно получить точную асимптотику малых уклонений для процесса Питмена $2 S-W$ во взвешенной норме.

Еще один интересный факт из [58, гл. 6, следствие 3.8] состоит в равенстве по распределению процессов $\left\{|W|(t)+L_{t}^{0}(W), t \geqslant 0\right\}$ и $\left\{\operatorname{Bes}^{3}(t), t \geqslant 0\right\}$. Отсюда вытекает точная асимптотика при $\varepsilon \rightarrow 0$ для вероятности $\mathbf{P}\left\{\left\||W|(t)+L_{t}^{0}(W)\right\|_{\psi} \leqslant \varepsilon\right\}$.

Распределение супремума по $x$ броуновского локального времени $L_{t}^{x}(W)$ хорошо изучено, его можно найти в [4, формула 1.11 .4$]$. Поэтому при $\varepsilon \rightarrow 0$ мы получаем

$$
\mathbf{P}\left\{\sup _{x \in \mathbf{R}} L_{t}^{x}(W) \leqslant \varepsilon\right\} \sim \frac{4}{\sin ^{2}\left(j_{0,1}\right)} e^{-2 j_{0,1}^{2} t / \varepsilon^{2}} .
$$

Интересно также посмотреть на распределение супремума броуновского локального времени вплоть до момента достижения уровня. Пусть $\tau_{b}=\inf \{s: W(s)=b\}-$ первый момент достижения уровня $b \in \mathbf{R}$. Тогда из [3, гл. I, формула (4.13)], см. также [4, формула (2.11.2)], вытекает следующая точная асимптотика при $\varepsilon \rightarrow 0$ :

$$
\mathbf{P}\left\{\sup _{-\infty<x<b} L_{\tau_{b}}^{x}(W) \leqslant \varepsilon\right\} \sim \frac{8}{j_{0,1}^{3} J_{1}\left(j_{0,1}\right)} e^{-b j_{0,1}^{2} / \varepsilon^{2}} .
$$


7. О малых уклонениях броуновского меандра. В этом разделе мы рассмотрим броуновский меандр (или извилину) $\mathfrak{m}$. Строгое определение броуновского меандра длины 1 имеется в [58, гл. XII] и [4, c. 83]. Этот процесс можно на неформальном уровне представлять себе как броуновское движение, подчиненное условию положительности вплоть до момента 1, однако не требуется, чтобы броуновский меандр обращался в нуль в точке 1 . Обозначим $\mathfrak{m}^{z}(t)$ броуновский меандр, принимающий значение $z \geqslant 0$ в момент времени 1 .

Следующий факт доказан в [28]:

$$
\left(\mathfrak{m}^{z}(t)\right)^{2} \stackrel{\text { law }}{=} B_{1}^{2}(t)+B_{2}^{2}(t)+\left(B_{3}(t)+z t\right)^{2}, 0 \leqslant t \leqslant 1,
$$

где $B_{1}, B_{2}$ и $B_{3}$ - это три независимых броуновских моста. При $z=0$ мы получаем в качестве частного случая тождество Леви-Уильямса, рассмотренное выше.

Сначала мы найдем точную асимптотику малых уклонений процесса $(B(t)+z t)^{2}$. Для этого можно использовать работу [8], в которой доказано, что при $r \rightarrow 0$

$$
\mathbf{P}\left\{\int_{0}^{1}(B(t)+z t)^{2} d t \leqslant r\right\} \sim \sqrt{\frac{8}{\pi\left(1+z^{2}\right)}} \exp \left(-\frac{\left(z^{2}+1\right)^{2}}{8 r}+\frac{z^{2}}{2}\right) .
$$

По формуле (20) мы имеем при $r \rightarrow 0$ :

$$
\mathbf{P}\left\{\left\|B_{1}\right\|^{2}+\left\|B_{2}\right\|^{2} \leqslant r\right\} \sim \frac{2 \sqrt{2}}{\sqrt{\pi r}} \exp \left(-\frac{1}{2 r}\right) .
$$

Комбинируя этот результат с (31), с помощью леммы 2 получаем, что при $\varepsilon \rightarrow 0$

$$
\begin{aligned}
\mathbf{P}\left\{\int_{0}^{1}\left(B_{1}^{2}(t)+B_{2}^{2}(t)+\left(B_{3}(t)+z t\right)^{2}\right) d t \leqslant \varepsilon^{2}\right\} \\
\\
\sim \frac{2 \sqrt{2\left(z^{2}+3\right)}}{\sqrt{\pi}} e^{z^{2} / 2} \varepsilon^{-2} \exp \left(-\frac{\left(z^{2}+3\right)^{2}}{8} \varepsilon^{-2}\right) .
\end{aligned}
$$

Итак, мы получили следующую точную асимптотику малых уклонений для броуновского меандра с предписанным концом $z$.

Предложение 9. Для любого $z \geqslant 0$, при $\varepsilon \rightarrow 0$ справедливо соотношение

$$
\mathbf{P}\left\{\left\|\mathfrak{m}^{z}\right\| \leqslant \varepsilon\right\} \sim \frac{2 \sqrt{2\left(z^{2}+3\right)}}{\sqrt{\pi}} \varepsilon^{-2} \exp \left(-\frac{\left(z^{2}+3\right)^{2}}{8} \varepsilon^{-2}+\frac{z^{2}}{2}\right) .
$$

При $z=0$ этот результат находится в прекрасном соответствии с формулой (29) для броуновской экскурсии. 
Переходим к малым уклонениям обычного броуновского меандра $\mathfrak{m}$. Для этого используем тождество по распределению из $[52$, следствие 3.9 .1$]$ или $[56, \S 5]$ :

$$
\left\{\mathfrak{m}^{2}(t), 0 \leqslant t \leqslant 1\right\} \stackrel{\text { law }}{=}\left\{B^{2}(t)+W_{1}^{2}(t)+W_{2}^{2}(t), 0 \leqslant t \leqslant 1\right\},
$$

где броуновский мост $B$ и два броуновских движения $W_{1}$ и $W_{2}$ независимы. Процесс в правой части (32) - это «интерполяция» между квадратами бесселевского процесса и бесселевского моста $[56, \S 5]$. С помощью (32) легко выводится точная асимптотика малых уклонений для меандра. Используя лемму 4 в частном случае $k=2$ и $m=1$, получаем следующий результат.

Предложение 10. При $\varepsilon \rightarrow 0$ справедливо соотношение

$$
\mathbf{P}\{\|\mathfrak{m}\| \leqslant \varepsilon\} \sim 4 \sqrt{\frac{2}{3 \pi}} \exp \left(-\frac{9}{8} \varepsilon^{-2}\right) .
$$

Аналогичным образом можно получить точную асимптотику малых уклонений для броуновского меандра с различными весами.

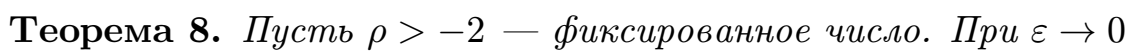

$$
\begin{aligned}
\mathbf{P}\left\{\int_{0}^{1} t^{\rho} \mathfrak{m}^{2}(t) d t \leqslant \varepsilon^{2}\right\} \sim & \frac{2^{2+\rho /(2(\rho+2))} \pi^{1 / 2}}{3^{1 / 2+3 \rho /(4(\rho+2))} \Gamma(1 /(\rho+2)) \Gamma^{1 / 2}((\rho+3) /(\rho+2))} \\
& \times((\rho+2) \varepsilon)^{3 \rho /(2(\rho+2))} \exp \left(-\frac{9}{4}((\rho+2) \varepsilon)^{-2}\right) .
\end{aligned}
$$

Для правильного веса $\psi$ на $[0,1]$

$$
\mathbf{P}\left\{\|\mathfrak{m}\|_{\psi} \leqslant \varepsilon\right\} \sim 4 \sqrt{\frac{2}{3 \pi}} \frac{\psi^{3 / 8}(0)}{\vartheta^{1 / 2} \psi^{1 / 8}(1)} \exp \left(-\frac{9 \vartheta^{2}}{8} \varepsilon^{-2}\right),
$$

где $\vartheta=\int_{0}^{1} \sqrt{\psi(t)} d t$.

Имеется интересное тождество по распределению, связывающее броуновский меандр $\mathfrak{m}(s)$ с броуновским мостом $B(s)$ и его локальным временем в нуле $L_{s}^{0}(B)$. Оно выглядит так (см. $\left.[52, \S 8.3]\right)$ :

$$
\{\mathfrak{m}(s), s \leqslant 1\} \stackrel{\text { law }}{=}\left\{|B(s)|+L_{s}^{0}(B), s \leqslant 1\right\} .
$$

Комбинируя теорему 8 с этим тождеством, нетрудно получить точную асимптотику малых уклонений для нормы $\left\||B(s)|+L_{s}^{0}(B)\right\|_{\psi}$.

Обратимся к точной асимптотике малых уклонений для супремума броуновской экскурсии $\mu(\mathfrak{e})=\sup _{0 \leqslant u \leqslant 1} \mathfrak{e}(u)$. Обозначим $\mathscr{T}_{4}$ квадрат нормы четырехмерного бесселевского моста, т.е.

$$
\mathscr{T}_{4}=\int_{0}^{1}\left(\sum_{i=1}^{4} B_{i}^{2}(t)\right) d t
$$


где $B_{i}, i=1, \ldots, 4,-$ независимые броуновские мосты. По лемме 3

$$
\mathbf{P}\left\{\mathscr{T}_{4} \leqslant r\right\} \sim \frac{8 \sqrt{2}}{\sqrt{\pi r^{3}}} \exp \left(-\frac{2}{r}\right) .
$$

Известно, кроме того (см. [52, $\S 11.3])$, что $\mu^{2}(\mathfrak{e}) \stackrel{\text { law }}{=}\left(\pi^{2} / 4\right) \mathscr{T}_{4}$. Комбинируя эти соотношения, получаем простое, но новое соотношение.

Предложение 11. При $\varepsilon \rightarrow 0$

$$
\mathbf{P}\{\mu(\mathfrak{e}) \leqslant \varepsilon\} \sim \frac{\pi^{2} \sqrt{2 \pi}}{\varepsilon^{3}} \exp \left(-\frac{\pi^{2}}{2 \varepsilon^{2}}\right) .
$$

Другим путем эта асимптотика может быть получена с помощью леммы 2 из знаменитого тождества Чжуна (см. $[52, \S 11.3]) \mu^{2}(\mathfrak{e}) \stackrel{\text { law }}{=}$ $\mu^{2}\left(\left|B_{1}\right|\right)+\mu^{2}\left(\left|B_{2}\right|\right)$, где независимые с.в. $\mu\left(\left|B_{1}\right|\right)$ и $\mu\left(\left|B_{2}\right|\right)$ имеют распределение Колмогорова. Это значит, что при всех $r>0$

$$
\mathbf{P}\{\mu(|B|) \leqslant r\}=\frac{\sqrt{2 \pi}}{r} \sum_{k=1}^{\infty} \exp \left(-\frac{(2 k-1)^{2} \pi^{2}}{8 r^{2}}\right) .
$$

Поэтому, при $r \rightarrow 0$

$$
\mathbf{P}\{\mu(|B|) \leqslant r\} \sim \frac{\sqrt{2 \pi}}{r} \exp \left(-\frac{\pi^{2}}{8 r^{2}}\right),
$$

и нам остается применить лемму 2.

Предложение 11 влечет за собой интересное соотношение, связанное с интегральным функционалом $h(\mathfrak{e})=\int_{0}^{1} d s / \mathfrak{e}(s)$.

Предложение 12.

$$
\mathbf{P}\{h(\mathfrak{e}) \leqslant \varepsilon\} \sim \frac{8 \pi^{2} \sqrt{2 \pi}}{\varepsilon^{3}} \exp \left(-\frac{2 \pi^{2}}{\varepsilon^{2}}\right), \quad \varepsilon \rightarrow 0 .
$$

Доказательство следует из тождества по распределению (см. [52, $\S 11.5])$ :

$$
h(\mathfrak{e}) \stackrel{\text { law }}{=} 2 \mu(\mathfrak{e}) .
$$

Еще одна асимптотика может быть выведена из (33). Пользуясь тождеством по распределению (см. [56, с. 250]):

$$
\int_{0}^{1}\left(\operatorname{Bes}_{0}^{2}(s)\right)^{-1} d s \stackrel{\text { law }}{=} 2 \mu(|B|),
$$

мы получаем формулу, напоминающую (24): при $r \rightarrow 0$

$$
\mathbf{P}\left\{\int_{0}^{1}\left(\operatorname{Bes}_{0}^{2}(s)\right)^{-1} d s \leqslant r\right\} \sim \frac{2 \sqrt{2 \pi}}{r} \exp \left(-\frac{\pi^{2}}{2 r^{2}}\right) .
$$


Интересно сравнить асимптотику малых уклонений для супремумов броуновской экскурсии и броуновского меандра. Известно тождество (см. [43], [30, с. 449]) $\mu(\mathfrak{m}) \stackrel{\text { law }}{=} 2 \mu(|B|)$, где правая часть совпадает с правой частью (35). Тогда, применив (33), получаем следующее соотношение.

Предложение 13.

$$
\mathbf{P}\{\mu(\mathfrak{m}) \leqslant \varepsilon\} \sim \frac{2 \sqrt{2 \pi}}{\varepsilon} \exp \left(-\frac{\pi^{2}}{2 \varepsilon^{2}}\right), \quad \varepsilon \rightarrow 0 .
$$

Сравнение предложений 11 и 13 показывает совпадение экспонент и различие в степенных членах и константах, как и можно было ожидать.

Интересно, что для броуновского меандра тождество (34) принимает похожий, но иной вид $[29]: h(\mathfrak{m}) \stackrel{\text { law }}{=} \mu(\mathfrak{m})$. Это позволяет, опираясь на предложение 13, вывести точную асимптотику малых уклонений для функционала $h(\mathfrak{m})=\int_{0}^{1} d s / \mathfrak{m}(s)$.

Авторы признательны профессорам А. Н. Бородину, М. А. Лифшицу и А. И. Назарову за ценные советы и предложения по улучшению работы.

\section{СПИСОК ЛИТЕРАТУРЫ}

1. Аурзада Ф., Ибрагимов И. А., Лифиии М. А., ван Зантен Х. Малые уклонения гладких стационарных гауссовских процессов. - Теория вероятн. и ее примен., 2008, т. 53 , в. 4 , с. $788-798$.

2. Бейтмен Г., Эрдейи А. Высшие трансцендентные функции, т. 2. М.: Наука, 1974, $296 \mathrm{c}$.

3. Бородин А. Н. Броуновское локальное время. - Успехи матем. наук, 1989, т. 44, B. 2 , c. $7-48$.

4. Бородин А.Н., Салминен П. Справочник по броуновскому движению. Факты и формулы. СПб: Лань, 2000, 640 с.

5. Гихман И. И. О непараметрическом критерии однородности $k$ выборок. - Теория вероятн. и ее примен., 1957 , т. 2, в. 2, с. 380-384.

6. Ибрагимов И.А. О вероятности попадания гауссова вектора со значениями в гильбертовом пространстве в сферу малого радиуса. - Зап. научн. сем. ЛОМИ, 1979 , т. 85 , с. $75-93$.

7. Лифиич М. А. Гауссовские случайные функции. Киев: ТВіМС, 1995, 246 с.

8. Михайлова E. М. Асимптотические распределения для броуновского движения со сносом. - Успехи матем. наук, 1994, т. 49, в. 4, с. 173-174.

9. Назаров А.И. О точной константе в асимптотике малых уклонений в $L_{2}$-норме некоторых гауссовских процессов. - Нелинейные уравнения и математический анализ. Новосибирск: Т. Рожковская, 2003, с. 179-214. (Проблемы матем. анализа, в. 26).

10. Назаров А.И., Пусев Р. С. Точная асимптотика малых уклонений в $L_{2}$-норме с весом для некоторых гауссовских процессов. - Зап. научн. сем. ПОМИ, 2009, т. 364 , с. $166-199$.

11. Никитин Я.Ю., Харинский П. А. Точная асимптотика малых уклонений в $L_{2}$ норме для одного класса гауссовских процессов. - Зап. научн. сем. ПОМИ, 2004, т. 311 , c. $214-221$.

12. Пусев P. С. Асимптотика малых уклонений процессов Боголюбова в квадратичной норме. - Теор. и мат. физика, 2010, т. 165, № 1, с. 134-144. 
13. Санкович Д. П. Гауссовы функциональные интегралы и гиббсовские равновесные средние. - Теор. и мат. физика, 1999, т. 119, № 2, с. 345-352.

14. Санкович Д. П. Функциональный интеграл Боголюбова. - Нелинейная динамика, сб. статей, Тр. МИАН, т. 251. М.: Наука, 2005, с. 223-256.

15. Справочник по специальным функциям с формулами, графиками и математическими таблицами. Под ред. М. Абрамовица и И. Стиган. М.: Наука, 1979.

16. Сытая Г. Н. О некоторых асимптотических представлениях гауссовской меры в гильбертовом пространстве. - Теория случайных процессов, 1974, т. 2, с. 93-104.

17. Титчмари E. Теория функций. М.: Наука, 1980, 463 с.

18. Фаталов B.P. Константы в асимптотиках вероятностей малых уклонений для гауссовских процессов и полей. - Успехи матем. наук, 2003, т. 58, № 4, с. 89-134.

19. Фаталов В.Р. Времена пребывания и точные асимптотики малых уклонений бесселевских процессов для $L^{p}$-норм, $p>0$. - Изв. РАН. Сер. матем., 2007, т. 71 , № 4, с. $69-102$.

20. Фаталов В.Р. Некоторые асимптотические формулы для гауссовской меры Боголюбова. - Теор. и мат. физика, 2008, т. 157, № 2, с. 286-308.

21. Фаталов В.Р. Точные асимптотики малых уклонений для стационарного процесса Орнштейна-Уленбека и некоторых гауссовских диффузий в $L^{p}$-норме, $2 \leqslant$ $p \leqslant \infty$. - Пробл. передачи информ., 2008, т. 44, в. 2, с. 75-95.

22. Федорюк М.В. Асимптотические методы для линейных обыкновенных дифференциальных уравнений. М.: Наука, 1983, 352 с.

23. Adler R.J. An introduction to continuity, extrema and related topics for general Gaussian processes. - Inst. of Math. Stat. Lect. Notes, Monograph Ser. 12. Inst. of Math. Stat., Hayward, CA, 1990, 160 p.

24. Alili L. On some hyperbolic principal values of Brownian local times. - Exponential Functionals and Principal Values Related to Brownian Motion (M. Yor, ed.) Biblioteca de la Revista Matematica Ibero-Americana, Madrid, 1997, p. 131-154.

25. Alili L., Donati-Martin C., Yor M. Une identité en loi remarquable pour l'excursion brownienne normalisée. - Exponential Functionals and Principal Values Related to Brownian Motion (M. Yor, ed.). Biblioteca de la Revista Matematica IberoAmericana, Madrid, 1997, p. 155-180.

26. Anderson T. W. Darling D. A. Asymptotic theory of certain «goodness-of-fit» criteria based on stochastic processes. - Ann. Mathem. Statist., 1952, v. 23, p. 193-212.

27. Beghin L., Nikitin Ya., Orsingher E. Exact small ball constants for some Gaussian processes under $L_{2}$-norm. - Зап. научн. сем. ПОМИ, 2003, т. 298, с. 5-21.

28. Bertoin J., Pitman J., Ruiz de Chavez J. Constructions of a Brownian path with a given minimum. - Electr. Commun. in Probab., 1999, v. 4, p. 31-37.

29. Biane Ph., Yor M. Valeurs principales associés aux temps locaux browniens. - Bull. Sci. Math., 2e série, 1987, v. 111, p. 23-101.

30. Biane Ph., Pitman J., Yor M. Probability laws related to the Jacobi theta and Riemann zeta functions, and Brownian excursions. - Bull. Amer. Math. Soc., 2001, v. 38 , № 4 , p. $435-465$.

31. Ciesielski Z., Taylor S. J. First passage times and sojourn times for Brownian motion in space and the exact Hausdorff measure of the sample path. - Trans. Amer. Math. Soc., 1962, v. 103, p. 434-450.

32. Коддингтон Э. А., Левинсон $Н$. Теория обыкновенных дифференциальных уравнений. М.: ИЛ, 1958, 474 с.

33. Csörgö M., Shi Z., Yor M. Some asymptotic properties of the local time of the uniform empirical process. - Bernoulli, 1999, v. 5, p. 1035-1058.

34. Dudley R. M., Hoffmann-Jørgensen J., Shepp L.A. On the lower tail of Gaussian seminorms. - Ann. Probab., 1979, v. 7, p. 319-342.

35. Dunker T., Lifshits M.A., Linde W. Small deviations of sums of independent variables. In: High Dimensional Probability. Progress in Probability, vol. 43, Birkhaeuser, Basel, 1998, pp. 59-74.

36. Ferraty F., Vieu Ph. Nonparametric Functional Data Analysis. Berlin: Springer, 2006, $258 \mathrm{p}$.

37. Fröman N., Fröman P. O. JWKB Approximation. Amsterdam: North-Holland, 1965.

38. Gao F., Hannig J., Lee T.-Y., Torcaso F. Laplace transforms via Hadamard 
factorization. - Electron. J. Probab., 2003, v. 8, № 13, p. 1-20.

39. Gao F., Hannig J., Lee T.-Y., Torcaso F. Exact $L_{2}$-small balls of Gaussian processes. - J. Theoret. Probab., 2004, v. 17, № 2, p. 503-520.

40. Gutierrez Jaimez R., Valderrama Bonnet M. J. On the Karhunen-Loève expansion for transformed processes. - Trab. Estad., 1987, v. 2, p. 81-90.

41. Ito K., McKean H.P. Diffusion Processes And Their Sample Paths. Berlin: Springer, $1965,321 \mathrm{p}$.

42. Janson S. Brownian excursion area, Wright's constants in graph enumeration, and other Brownian areas. - Probability Surveys, 2007, v. 4, p. 80-145.

43. Kennedy D. P. The distribution of the maximum Brownian excursion. - J. Appl. Probab., 1976, v. 13, p. 371-376.

44. Kiefer J. $k$-sample analogues of the Kolmogorov-Smirnov and Cramér-von Mises tests. - Ann. Math. Stat., 1959, v. 30, p. 420-447.

45. Lévy P. Processus stochastiques et mouvement brownien. Paris: Gauthier-Villars, 1965.

46. $L i W . V$. Comparison results for the lower tail of Gaussian seminorms. - J. Theoret. Probab., 1992, v. 5, № 1, p. 1-31.

47. $L i W . V$., Shao Q. M. Gaussian processes: inequalities, small ball probabilities and applications. - Stochastic Processes: Theory and Methods. Amsterdam: NorthHolland, 2001, p. 533-597. (Handbook Statist., v. 19.)

48. Lifshits M. A. On the lower tail probabilities of some random series. - Ann. Probab., 1997 , v. 25 , № 1 , p. 424-442.

49. Lifshits M. A. Asymptotic behavior of small ball probabilities. - Proceedings of the Seventh International Vilnius conference on Probability Theory and Mathematical Statistics. Utrecht/Vilnius: VSP/TEV, 1999, p. 453-468.

50. Lifshits M.A. Bibliography on small deviation probabilities. http://www.proba.jussieu.fr/pageperso/smalldev/biblio.pdf.

51. Mansuy $R$. An interpretation and some generalizations of the Anderson-Darling statistics in terms of squared Bessel bridges. - Statistics and Probab. Lett., 2005, v. 72 , p. $171-177$.

52. Mansuy R., Yor M. Aspects of Brownian Motion. Berlin: Springer-Verlag, 2008, 195 p.

53. Nazarov A.I., Nikitin Ya. Yu. Exact $L_{2}$-small ball behavior of integrated Gaussian processes and spectral asymptotics of boundary value problems. - Probab. Theory Related Fields, 2004, v. 129, № 4, p. 469-494.

54. Nazarov A. I. Exact $L_{2}$-small ball asymptotics of Gaussian processes and the spectrum of boundary-value problems. - J. Theoret. Probab., 2009, v. 22, p. 640-665.

55. Pitman J. One-dimensional Brownian motion and the three-dimensional Bessel process. - Adv. Appl. Prob., 1975, v. 7, p. 511-526.

56. Pitman J., Yor M. Quelques identités en loi pour les processus de Bessel. Astérisque, 1976 , v. 236, p. 249-276.

57. Pitman J., Yor M. The law of the maximum of a Bessel bridge. - Electron. J. Probab., 1999, v. 4, p. 1-35.

58. Revuz D., Yor M. Continuous Martingales and Brownian Motion. 2nd ed. Berlin: Springer, 2001, $602 \mathrm{p}$

59. Rogers L.C.G., Williams D. Diffusions, Markov Processes and Martingales, v. 2. Cambridge: Cambridge University Press, 2000, 494 p.

60. van der Vaart $A$. W., van Zanten $H$. Rates of contraction of posterior distributions based on Gaussian process priors. - Ann. Statist., 2008, v. 36, № 3, p. 1435-1463.

61. Williams D. Decomposing the Brownian path. - Bull. Amer. Math. Soc., 1970, v. 76, p. 871-873.

62. Zolotarev V. M. Gaussian measure asymptotics in $l_{2}$ on a set of centered spheres with radii tending to zero. - 12th Europ. Meeting of Statisticians, Varna, 1979, p. 254.

Поступила в редакцию 18.XII.2011 